\title{
Chronic kidney disease with genitourinary tuberculosis: old disease but ongoing complication
}

\author{
Eun Jin Kim', Woonji Lee ${ }^{2}$, Woo Yong Jeong ${ }^{2,3}$, Hen Choi ${ }^{2,3}$, In Young Jung 2,3, Jin Young Ahn ${ }^{2,3}$, Su Jin Jeong ${ }^{2,3}$, \\ Nam Su Ku ${ }^{2,3^{*}+} \mathbb{D}$, Jun Yong Choi ${ }^{2,3}$, Young Hwa Choi ${ }^{{ }^{* \dagger}}$, Young Goo Song ${ }^{2,3}$ and June Myung Kim ${ }^{2,3}$
}

\begin{abstract}
Background: Genitourinary tuberculosis (GUTB) is a type of extrapulmonary TB that exerts a deleterious effect on renal function by promoting renal calcification and ureteric stricture. Therefore, we investigated the risk factors for chronic kidney disease (CKD) in GUTB patients after the end of treatment.

Methods: This retrospective study was conducted at a tertiary hospital in South Korea. Data from patients (>18 years of age) with GUTB were collected from January 2005 to July 2016. CKD was defined as a glomerular filtration rate $<60 \mathrm{~mL} / \mathrm{min} / 1.73 \mathrm{~m}^{2}$ after the end of treatment.

Results: In total, 56 patients with GUTB (46.4\% males; mean age $52.8 \pm 16.6$ years) were enrolled in the study. CKD developed in 11 (19.6\%) patients and end-stage renal disease in 4 (7.1\%). In a univariate analysis, older age ( $p=0$. 029), microscopic haematuria $(p=0.019)$, proteinuria $(p=0.029)$, acute renal failure (ARF) $(p<0.001)$ and a positive polymerase chain reaction-based test result for TB in the urine $(p=0.030)$ were significantly associated with decreased renal function. In a multivariate analysis, ARF (odds ratio [OR], 54.31; 95\% confidence interval [Cl], $1.52-$ $1944.00 ; p=0.032)$ and old age $(\mathrm{OR}, 54.26 ; 95 \% \mathrm{Cl}, 1.52-1932.94 ; p=0.028)$ were independent risk factors for CKD in GUTB patients.

Conclusions: ARF and old age were independent risk factors for CKD in GUTB patients. Therefore, in elderly GUTB patients with ARF at the time of diagnosis, regular follow-up of renal function should be performed even after the end of treatment.
\end{abstract}

Keywords: Genitourinary tuberculosis, Chronic kidney disease, Risk factor

\section{Background}

Tuberculosis (TB) is an important unresolved public health issue, affecting millions of people each year. In 2015 , TB was one of the top 10 infectious causes of death worldwide; there were 10.4 million new TB cases, equivalent to 142 cases per 100,000 population, and 1.4 million deaths due to $\mathrm{TB}$, with an additional 0.4 million deaths

\footnotetext{
* Correspondence: smileboy9@yuhs.ac; yhwa1805@ajou.ac.kr ${ }^{+} \mathrm{Nam}$ Su Ku and Young Hwa Choi contributed equally to this work. ${ }^{2}$ Division of Infectious Disease, Department of Internal Medicine, Yonsel University College of Medicine, 50-1 Yonsei-ro, Seodaemun-gu, Seoul 120-752, Republic of Korea

'Department of Infectious Diseases, Ajou University School of Medicine, 164, World cup-ro, Yeongtong-gu, Suwon-si, Gyeonggi-do 16499, Republic of Korea

Full list of author information is available at the end of the article
}

resulting from $\mathrm{TB}$ among human immunodeficiency virus (HIV)-positive persons [1]. South Korea has an intermediate burden of TB; according to the Korea Center for Disease Control, the estimated TB incidence is 63.2 cases per 100,000 population (in $2015, n=32,181$ ). Extrapulmonary TB (EPTB) accounted for $13.0 \%$ of all new cases reported in $2015(n=6631)$. Genitourinary TB (GUTB) is a type of EPTB that infected 199 individuals in South Korea in 2015, accounting for 3.0\% of all EPTB cases [2, 3].

Although GUTB is a rare form of EPTB, it is an important cause of progressive chronic kidney disease (CKD). The kidneys are the most common site of GUTB, with bacteria spreading haematogenously. Tuberculous bacilli can lead to granuloma formation in glomeruli and entry into the medullary interstitium. Subsequently, renal

(c) The Author(s). 2018 Open Access This article is distributed under the terms of the Creative Commons Attribution 4.0 International License (http://creativecommons.org/licenses/by/4.0/), which permits unrestricted use, distribution, and reproduction in any medium, provided you give appropriate credit to the original author(s) and the source, provide a link to the Creative Commons license, and indicate if changes were made. The Creative Commons Public Domain Dedication waiver (http://creativecommons.org/publicdomain/zero/1.0/) applies to the data made available in this article, unless otherwise stated. 
papilla destruction can develop due to calyceal ulceration and involvement of the collecting system. This destruction may extend towards the urothelium and induce stricture formation, resulting in hydroureter and hydronephrosis. Renal calcification in GUTB is common, and patients with renal TB can develop bladder contracture. In addition, TB can affect the male and female genital tracts $[4,5]$. GUTB induces end-stage renal disease (ESRD) in 5.7\% of patients [5]. According to the European Dialysis and Transplant Association registry, $0.65 \%$ of new dialysis cases are caused by renal TB [6]. In Korea, there were 14,756 new dialysis patients in 2015 , of whom $0.1 \%$ required dialysis because of GUTB [7, 8].

However, in a previous autopsy study, only $50 \%$ of patients with GUTB were symptomatic, and only $18 \%$ had received a clinical diagnosis [5]. Additionally, the clinical manifestations of GUTB are nonspecific [9]. Thus, the diagnosis is often delayed, during which GUTB progression may lead to CKD due to parenchymal destruction and obstructive uropathy. Therefore, we investigated the characteristics of urogenital TB in adult patients with no history of CKD in a single-centre retrospective observational study and identified risk factors for CKD development after ending GUTB treatment.

\section{Methods}

\section{Study population and design}

We conducted this retrospective study at the Severance Hospital, a 2400-bed university-affiliated teaching hospital and tertiary care referral hospital in Seoul, South Korea. We enrolled participants older than 18 years of age diagnosed with GUTB. We identified patients with ICD-10 codes A18.1, B90.1 and N33.0 from January 2005 to July 2016 from electronic medical records and enrolled only those who started and completed the treatment during the study period. Clinical and laboratory data at the time of GUTB diagnosis, including age, sex, medical history, follow-up duration and symptoms, were collected. To assess renal function, serum creatinine levels were reported before treatment and at the 6- and 12-month post-treatment follow-ups. Body mass index (BMI) was calculated as weight divided by height squared $\left(\mathrm{kg} / \mathrm{m}^{2}\right)$. We also investigated the diagnostic methods, treatment modalities and outcomes of GUTB patients during a recent 10 -year period in one centre in Korea to identify risk factors for CKD developing after ending treatment. All surgical techniques were investigated included all ablative surgery and reconstructive surgery between diagnosis and during medical treatment. We excluded patients with a follow-up duration of $<1$ year, unfulfilled GUTB diagnostic criteria, pre-existing CKD and insufficient data. This study was approved by the Institutional Review Board of the Yonsei University Health System Clinical Trial Center.

\section{Definitions}

The diagnosis of GUTB was defined as the presence of any clinical finding plus a positive result for one of the following: (1) acid-fast bacilli (AFB) in urine, (2) urine culture of Mycobacterium tuberculosis (M. tuberculosis), (3) polymerase chain reaction (PCR) for $M$. tuberculosis in urine or (4) histopathological evidence of TB in any GU tissue specimen. A histological diagnosis of TB was confirmed by identifying caseating necrosis, loose aggregates of epithelioid histiocytes and Langerhans giant cells in tissue specimens $[6,10]$. Immunosuppressant use was defined as a daily dose of $\geq 20 \mathrm{mg}$ prednisolone-equivalent steroid, monoclonal antibodies, antimetabolite drugs or T-cell inhibitors within 30 days prior to diagnosis of GUTB. Pre-TB (pre-existing) CKD was defined as an estimated glomerular filtration rate (eGFR) of $<60 \mathrm{~mL} / \mathrm{min} /$ $1.73 \mathrm{~m}^{2}$ for more than 3 months before diagnosis of GUTB or a self-reported history. Acute renal failure (ARF) was defined according to the KDIGO (Kidney Disease: Improving Global Outcomes) criteria (increased serum creatinine level $\geq 1.5$-fold compared with baseline or by $\geq 0.3 \mathrm{mg} / \mathrm{dL}$ ). We used the serum creatinine level to investigate the presence of ARF at the time of GUTB diagnosis. The eGFR was calculated from the serum creatinine level using the Modification of Diet in Renal Diseases (MDRD) equation [11]. Microscopic haematuria was defined as the excretion of more than two red blood cells per high-power field in a centrifuged urine specimen and pyuria as the excretion of more than five white blood cells. Proteinuria was defined as more than one positive urine dipstick test. Anaemia was defined as a haemoglobin level $<11 \mathrm{~g} / \mathrm{dL}$ according to the KDIGO guidelines based on the target for renal anaemia therapy [12].

\section{Decreased kidney function and poor outcome}

CKD group was defined as an eGFR $<60 \mathrm{~mL} / \mathrm{min} / 1.73 \mathrm{~m}^{2}$ after completing GUTB treatment. We used the serum creatinine level between 6 months and 1 year after completion of GUTB treatment to investigate the presence of CKD. Patients with a history of CKD at the time of GUTB diagnosis (pre-TB CKD) were excluded. To assess kidney function, serum creatinine levels were determined before treatment and at the 6- and 12-month post-treatment follow-ups. After the treatment was completed, any surgical treatment, recurrence, ESRD development, and all cause mortality were investigated.

\section{Statistical analysis}

All statistical analyses were performed using the Statistical Package for the Social Sciences for Windows (ver. 23.0, SPSS Inc., Chicago, IL, USA). To identify risk factors for CKD, parameters were compared between patients who did and those who did not develop CKD. Continuous variables are expressed as means \pm standard deviation and 
categorical variables as numbers (percentages). The Kolmogorov-Smirnov test was used to analyse the normality of the data distribution. Non-normally distributed data are expressed as medians and interquartile ranges (IQRs). We used Student's $t$-test or Mann-Whitney U-test and the $\chi^{2}$ test or Fisher's exact test to compare continuous and categorical variables, respectively, in univariate analyses. Variables with $p$-values $<0.05$ by the Wald test in the univariate analyses were included in a multivariate logistic regression analysis to identify risk factors for CKD. The multivariate analysis results are expressed as odds ratios (ORs) and 95\% confidence intervals (CIs). A two-sided $p$ value $<0.05$ was considered to indicate significance.

\section{Results}

\section{Demographic characteristics}

During a 10-year period, 56 patients were diagnosed with GUTB, of whom 11 (19.6\%) developed CKD after treatment for GUTB (Fig. 1). The mean age of these patients was 52.8 years, and $24(42.9 \%)$ patients had a history of TB, most frequently pulmonary TB (21/24). Three patients were taking immunosuppressants, and two had a history of gastrectomy. No patient had HIV or multi-drug resistant TB. The most frequent symptom was abdominal pain (42.9\%), and gross haematuria was present in 33\% of the patients (Table 1).

The diagnosis was confirmed most frequently by histopathology (30/56, 53.6\%). Urine AFB staining was positive in six of the 56 patients (10.7\%). A TB interferon-gamma evaluation was performed in $5 / 56$ patients, all of whom showed a positive result.

Almost all of the patients $(53 / 56,94.6 \%)$ underwent a 6or 9-month course of chemotherapy with isoniazid, rifampicin, and ethambutol with or without pyrazinamide; the median treatment duration was 9 months. In addition, 42 of the 56 patients $(75.0 \%)$ underwent surgical treatment. All surgical techniques included all ablative surgery and reconstructive surgery from the time of diagnosis to completion of medical treatment. During an average 40-month observation period, CKD developed in 11 (19.6\%) and ESRD in $4(7.1 \%)$ of the patients. Of the patients, $26.8 \%$ required additional surgical treatment after completion of medical TB treatment, and recurrence was observed in 4 (7.1\%) patients. The all-cause mortality rate was $7.1 \%(4 / 56)$.

\section{Risk factors for CKD}

CKD occurred in 11 (19.6\%) patients during the follow-up period. In univariate analyses, patients with CKD were older $(50.1 \pm 15.1$ vs. $63.9 \pm 18.5$ years; $p=0.012)$ and had higher creatinine levels at the time of diagnosis $(0.9$ [IQR $0.8-1.0]$ vs. 1.4 [IQR $1.1-1.8] \mathrm{mg} / \mathrm{dL} ; p=0.002)$. ARF occurred in 10 of the 56 patients (17.9\%), of whom three recovered and seven progressed to CKD due to persistent renal insufficiency.

There were significantly lower rates of microscopic haematuria $(42.2 \%$ vs. $81.8 \% ; p=0.019)$ and proteinuria $(13.3 \%$ vs. $45.5 \% ; p=0.029)$ in the non-CKD group compared with the CKD group in the univariate analyses. There was also a significantly higher rate of positive urine TB PCR results in the CKD group $(72.7 \%$ vs. $37.8 \% ; p=$ 0.030) (Table 2). In the CKD group, five patients had both microscopic haematuria and proteinuria, four had only microscopic haematuria, and two patients had neither microscopic haematuria nor proteinuria. Microscopic haematuria and proteinuria were significant (microscopic haematuria, $p=0.019$; proteinuria, $p=0.029$ ) in the univariate analyses but not in the multivariate analysis.

The sex ratio and BMI did not show significant differences between the two groups. The site of TB infection, previous TB history and comorbidities were not significant independent factors. Treatment modality and duration did not differ significantly between the two groups. Surgical treatment including nephrectomy may be performed during medical treatment, so it does not affect creatinine at

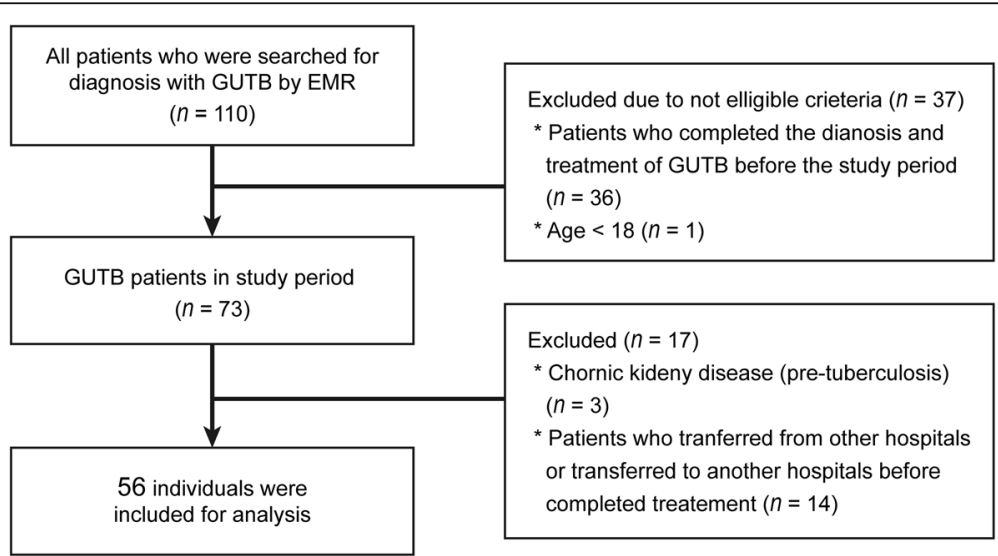

Fig. 1 Inclusion and exclusion criteria applied in this study. GUTB genitourinary tuberculosis, EMR electronic medical record 
Table 1 Baseline characteristics of both groups according to kidney function in patients with GUTB

\begin{tabular}{|c|c|c|c|c|}
\hline Variables & $\begin{array}{l}\text { Normal kidney function } \\
\text { group }(n=45)\end{array}$ & CKD group $(n=11)$ & Total $(n=56)$ & $p$ \\
\hline Age & $50.1 \pm 15.1$ & $63.9 \pm 18.5$ & $52.8 \pm 16.6$ & 0.012 \\
\hline Old age ( $\geq 65$ years) & $6(13.3)$ & $5(45.5)$ & 11 (19.6) & 0.029 \\
\hline Sex (male) & $20(44.4)$ & $6(54.5)$ & $26(46.4)$ & 0.609 \\
\hline BMI $\left(\mathrm{kg} / \mathrm{m}^{2}\right)$ & $22.8 \pm 3.4$ & $21.9 \pm 5.2$ & $22.6 \pm 3.8$ & 0.589 \\
\hline \multicolumn{5}{|l|}{ Previous TB history } \\
\hline Lung & $16(35.6)$ & $5(45.5)$ & $21(37.5)$ & 0.730 \\
\hline Bone & $2(4.4)$ & $1(9.1)$ & $3(5.4)$ & 0.488 \\
\hline Intestine & $1(2.2)$ & $0(0.0)$ & $1(1.8)$ & 1.000 \\
\hline \multicolumn{5}{|l|}{ Co-morbidity conditions } \\
\hline Immune-compromised patients & $8(17.8)$ & $4(36.4)$ & $12(21.4)$ & 0.224 \\
\hline Cardiovascular disease & $11(24.4)$ & $5(45.5)$ & $16(28.6)$ & 0.312 \\
\hline DM & $1(2.2)$ & $2(18.2)$ & $3(5.4)$ & 0.174 \\
\hline Liver disease & $3(6.7)$ & $1(9.1)$ & $4(7.1)$ & 1.000 \\
\hline Pulmonary disease & $4(8.9)$ & $2(18.2)$ & $6(10.7)$ & 0.727 \\
\hline History of gastrectomy & $2(4.4)$ & $0(0.0)$ & $2(3.6)$ & 1.000 \\
\hline \multicolumn{5}{|l|}{ Involving location } \\
\hline \multicolumn{5}{|l|}{ Urinary system } \\
\hline Kidney or ureter & $30(66.7)$ & $9(81.8)$ & 39 (69.6) & 0.539 \\
\hline Bladder & $11(24.4)$ & $5(45.5)$ & $16(28.6)$ & 0.312 \\
\hline \multicolumn{5}{|l|}{ Genital system } \\
\hline Epididymis or testis & $11(24.4)$ & $2(18.2)$ & $13(23.2)$ & 0.966 \\
\hline Prostate & $4(8.9)$ & $0(0.0)$ & $4(7.1)$ & 0.709 \\
\hline Uterus or fallopian tubes & $4(8.9)$ & $1(9.1)$ & $5(8.9)$ & 1.000 \\
\hline Both affected urinary and genital tract & $2(4.4)$ & $0(0.0)$ & $2(3.6)$ & 1.000 \\
\hline Concurrent extra-urogenital lesion & $1(2.2)$ & $1(9.1)$ & $2(3.6)$ & 0.132 \\
\hline \multicolumn{5}{|l|}{ Clinical features } \\
\hline Nonspecific symptoms ${ }^{a}$ & $5(11.1)$ & $2(18.2)$ & $7(12.5)$ & 0.614 \\
\hline Urinary symptoms ${ }^{b}$ & $22(48.9)$ & $9(81.8)$ & $31(55.4)$ & 0.088 \\
\hline Gross hematuria & $14(31.1)$ & $5(45.5)$ & 19 (33.9) & 0.481 \\
\hline Loin/abdominal pain & $18(40.0)$ & $6(54.5)$ & $24(42.9)$ & 0.501 \\
\hline Scrotal pain/mass & $11(24.4)$ & $0(0.0)$ & $11(19.6)$ & 0.098 \\
\hline Abscess or fistula & $2(4.4)$ & $1(9.1)$ & $3(5.4)$ & 0.488 \\
\hline Vaginal bleeding & $2(4.5)$ & $0(0.0)$ & $2(3.6)$ & 1.000 \\
\hline Asymptomatic patients & $4(8.9)$ & $0(0.0)$ & $4(7.1)$ & 0.575 \\
\hline
\end{tabular}

Bold values indicate statistically significant differences

Data were presented as mean \pm SD, number (percentage) or median (IQR)

GUTB genitourinary tuberculosis, CKD chornic kidney disease, BMI body mass index, TB tuberculosis, DM diabetes mellitus, SD standard deviation, IQR

interquartile range

${ }^{a} F e v e r ;$ anorexia/weight loss; sweating; weakness; peripheral lymphadenopathy

bUrinary frequency or dysuria; urethral pain; irritable voiding symptoms

the time of diagnosis but may affect CKD development. Of the patients, $15 / 56(26.8 \%)$ had total or partial nephrectomy; there were no significant differences between the two groups according to kidney function $(p=0.674)$. But, the rate of ESRD development $(0.0 \%$ vs. $36.4 \% ; p=0.001)$ and the rate of surgical treatment $(20.0 \%$ vs. $54.5 \% ; p=0.005)$ after completion of treatment were significantly higher in the CKD group than in the non-CKD group (Table 3).

In the multivariate analysis, ARF (OR, 54.305; 95\% CI, $1.517-1944.002 ; p=0.032$ ) and old age (OR, 54.255; 95\% CI, $1.523-1932.905 ; p=0.028)$ were independent risk factors for CKD in GUTB patients (Table 4). 
Table 2 Findings of both groups according to kidney function in patients with GUTB

\begin{tabular}{|c|c|c|c|c|}
\hline Variables & $\begin{array}{l}\text { Normal kidney function } \\
\text { group }(n=45)\end{array}$ & CKD group $(n=11)$ & Total $(n=56)$ & $p$ \\
\hline \multicolumn{5}{|l|}{ Diagnosis } \\
\hline Positivity of urine AFB stain & 3/41 (7.3) & $3 / 10(30.0)$ & 6/51 (11.8) & 0.081 \\
\hline Positivity of urinary TB culture & $17 / 38(44.7)$ & 7/10 (70.0) & $24 / 48(50.0)$ & 0.155 \\
\hline Positivity of urine TB PCR study & $17 / 36(47.2)$ & 8/9 (88.9) & 25/45 (55.6) & 0.030 \\
\hline Histopathologic diagnosis & $24(53.3)$ & $6(54.5)$ & 30/55 (53.6) & 0.741 \\
\hline \multicolumn{5}{|l|}{ Laboratory data } \\
\hline White blood cell $(/ \mu l)$ & $6880(5650-7890)$ & $6520(5580-8395)$ & 6695 (5580-7915) & 0.699 \\
\hline Hemoglobin (g/dl) & $12.5 \pm 1.8$ & $11.7 \pm 1.7$ & $12.3 \pm 1.8$ & 0.174 \\
\hline Platelet $\left(10^{3} / \mu \mathrm{l}\right)$ & $259.0 \pm 80.5$ & $263.2 \pm 121.2$ & $259.9 \pm 89.1$ & 0.893 \\
\hline ESR $(\mathrm{mm} / \mathrm{hr})$ & $46.9 \pm 23.8$ & $82.5 \pm 53.0$ & $53.4 \pm 30.7$ & 0.145 \\
\hline Blood urea nitrogen (mg/dl) & $13.9(10.5-16.9)$ & $17.8(15.4-27.8)$ & $14.4(11.6-17.6)$ & 0.006 \\
\hline Creatinine (mg/dl) & $0.9(0.8-1.0)$ & $1.4(1.1-1.8)$ & $1.0(0.8-1.1)$ & 0.002 \\
\hline eGFR (MDRD) & $78.1 \pm 16.5$ & $49.4 \pm 23.4$ & $75.8 \pm 23.5$ & 0.002 \\
\hline eGFR (MDRD) after 1 year & $82.7 \pm 16.5$ & $35.1 \pm 18.1$ & $72.8 \pm 25.6$ & $<0.001$ \\
\hline Acute renal failure & $3(6.7)$ & $7(63.6)$ & $10(17.9)$ & $<0.001$ \\
\hline Glucose (mg/dl) & $98.0(89.0-108.0)$ & $104.0(99.5-121.5)$ & $100.0(90.0-112.0)$ & 0.075 \\
\hline Albumin (g/dl) & $4.4(3.9-4.6)$ & $3.8(3.5-4.2)$ & $4.3(3.8-4.5)$ & 0.102 \\
\hline CRP (mg/dl) & $10.2(3.7-38.2)$ & $45.3(19.3-65.5)$ & $15.0(5.0-44.2)$ & 0.090 \\
\hline Microscopic hematuria & $19(42.2)$ & $9(81.8)$ & $28(50.0)$ & 0.019 \\
\hline Gross hematuria & $14(31.1)$ & $5(45.5)$ & 19 (33.9) & 0.481 \\
\hline Pyuria & $29(64.4)$ & $8(72.7)$ & $37(66.1)$ & 0.732 \\
\hline Proteinuria & $6(13.3)$ & $5(45.5)$ & 11 (19.6) & 0.029 \\
\hline
\end{tabular}

Bold values indicate statistically significant differences

Data were presented as mean \pm SD, number (percentage) or median (IQR). All data are based on the results at the time of diagnosis except for 'eGFR after 1 year' GUTB genitourinary tuberculosis, CKD chronic kidney disease, $A F B$ acid-fast bacilli, TB tuberculosis, $P C R$ polymerase chain reaction, ESR erythrocyte sedimentation rate, eGFR estimated glomerular filtration rate, MDRD Modification of Diet in Renal Disease study equation, $C R P$ c-reactive protein, $S D$ standard deviation, $I Q R$ interquartile range

\section{Discussion}

In this study, we report a high incidence of CKD after treatment in patients whose renal function was normal before the diagnosis of GUTB. Additionally, ARF and old age were independent risk factors for CKD in GUTB patients. This finding suggests that regular follow-up of renal function is needed during and after completion of treatment in elderly GUTB patients with an elevated creatinine level at the time of diagnosis.

CKD is a major global health issue and is ranked 17th in terms of disability-adjusted life years in the US [13]. The overall prevalence of CKD among the general population is $13.1 \%$ in the US and $10.8 \%$ in China $[14,15]$. In Korea, the prevalence of CKD among adults aged $>20$ years was $8.2 \%$, and that among urban civilians aged $>35$ years was $13.7 \%[16,17]$. In our study, the prevalence of CKD in GUTB patients was $19.6 \%$, higher than that in the general population. Likewise, a 19.0\% prevalence of renal function deterioration in GUTB patients was reported previously [10]. In our study, ESRD occurred in 7.1\% of GUTB patients. According to a review of 8961 cases, GUTB led to ESRD in $5.7 \%$ of patients [5]; that prior study involved patients aged 40-45 years, whereas our patients were older (mean, $52.8 \pm 16.6$ years), which may explain the higher incidence of ESRD [5, 18].

In this study, old age was an important risk factor for CKD in GUTB patients. The eGFR decreases in parallel with age [19]. Renal aging is a multifactorial process associated with anatomical and functional changes that accumulate over a lifetime [20]. Exposure to chronic inflammation, such as that induced by TB, likely enhances oxidative stress and endothelial dysfunction, which are related to renal aging. In addition, the renal-aging-induced reduction in kidney repair ability and tubular and glomerular changes aggravate the decrease in eGFR [20]. Elderly patients with GUTB require close monitoring for early detection of CKD.

A high creatinine level at the time of diagnosis reflects the progression of GUTB and destruction of the kidney structure. After treatment, recovery of a destroyed kidney is difficult. M. tuberculosis spreads from the kidney to the bladder, causing granulomatous lesions associated with fibrosis. In the kidney, M. tuberculosis forms granulomas in 
Table 3 Treatment modality and complications of both groups according to kidney function in GUTB patients

\begin{tabular}{|c|c|c|c|c|}
\hline Variables & $\begin{array}{l}\text { Normal kidney function } \\
\text { group }(n=45)\end{array}$ & CKD group $(n=11)$ & Total $(n=56)$ & $p$ \\
\hline \multicolumn{5}{|l|}{ Medical treatment } \\
\hline TB medication use & $42(93.3)$ & $11(100.0)$ & $53(94.6)$ & 0.894 \\
\hline Medication regimen & & & & 1.000 \\
\hline HER & $6(13.3)$ & $1(9.1)$ & $7(12.5)$ & \\
\hline HERZ & $36(80.0)$ & $10(90.9)$ & $46(82.1)$ & \\
\hline Medication durations (m) & $9(6-9)$ & $9(9-10)$ & $9(6-10)$ & 0.243 \\
\hline \multicolumn{5}{|l|}{ Surgical treatment } \\
\hline All surgical techniques ${ }^{a}$ & $32(71.1)$ & $10(90.9)$ & $42(75.0)$ & 0.332 \\
\hline Ablative surgery ${ }^{b}$ & $23(51.5)$ & $8(72.7)$ & $31(55.4)$ & 0.340 \\
\hline Nephrectomy & $11(24.4)$ & $4(36.4)$ & $15(26.8)$ & 0.674 \\
\hline Reconstructive therapy ${ }^{c}$ & $12(26.7)$ & $3(27.3)$ & $15(26.8)$ & 1.000 \\
\hline Follow up durations (m) & $38.2 \pm 27.8$ & $48.0 \pm 47.0$ & $40.5 \pm 32.5$ & 0.362 \\
\hline \multicolumn{5}{|l|}{ Complications } \\
\hline Hydronephrosis & $9(20.0)$ & $3(27.3)$ & $12(21.4)$ & 0.038 \\
\hline Bladder contraction & $3(6.7)$ & $2(18.2)$ & $5(8.9)$ & 0.021 \\
\hline Renal calcification & $10(22.2)$ & $3(27.3)$ & $13(23.2)$ & 0.044 \\
\hline Additional surgical treatment ${ }^{d}$ & $9(20.0)$ & $6(54.5)$ & $15(26.8)$ & 0.005 \\
\hline Recurrence & $3(6.7)$ & $1(9.1)$ & $4(7.1)$ & 0.056 \\
\hline All cause mortality & $2(4.4)$ & $2(18.2)$ & $4(7.1)$ & 0.132 \\
\hline ESRD development & $0(0.0)$ & $4(36.4)$ & $4(7.1)$ & 0.001 \\
\hline
\end{tabular}

Bold values indicate statistically significant differences

Data were presented as mean \pm SD, number (percentage) or median (IQR)

GUTB genitourinary tuberculosis, CKD chronic kidney disease, TB tuberculosis, HER isoniazid, rifampicin, and ethambutol, HERZ isoniazid, rifampicin, ethambutol, with pyrazinamide, ESRD end-stage renal disease, SD standard deviation, IQR interquartile range

${ }^{a}$ All surgical techniques included all ablative surgery and reconstructive surgery from the time of diagnosis to completion of medical treatment

${ }^{b}$ Ablative surgery is associated with partial or total nephrectomy, nephro-ureterectomy, cystectomy, epididymectomy, semicastration, salpingectomy, as well as other procedures

${ }^{\mathrm{C}}$ Reconstructive therapy is considered for: ureteric or urethral stricture repair; stent placement, replacement, or reimplantation; resection; urinary diversion; and bladder augmentation cystoplasty

${ }^{d}$ Any surgical treatment during follow up after completed medical treatment

the medullary region and can subsequently disseminate and destroy the renal parenchyma. Papillary necrosis eventually invades the collecting system, leading to fibrosis and obstructive uropathy [21, 22]. Throughout the disease course, papillary necrosis, tubular injury and obstructive uropathy can lead to CKD. In our study, ARF was significantly predictive of CKD and may be related to disease severity at the time of diagnosis. The 10 patients with ARF underwent ablative surgery or reconstruction because of direct infection of the kidney parenchyma and/or ureteral obstruction. Moreover, 10 of the 11 patients in whom CKD developed underwent ablative surgery or reconstruction because of direct infection of the kidney parenchyma and/or ureteral obstruction. Therefore, CKD can be caused by direct

Table 4 Multivariable analysis of risk factors associated chronic kidney disease in GUTB patients

\begin{tabular}{|c|c|c|c|c|}
\hline \multirow[t]{2}{*}{ Variables } & Univariate analysis & \multirow[t]{2}{*}{$p$} & Multivariable analysis & \multirow[t]{2}{*}{$p$} \\
\hline & OR $(95 \% \mathrm{Cl})$ & & OR $(95 \% \mathrm{Cl})$ & \\
\hline Old age ( $\geq 65$ years) & $5.42(1.25-23.45)$ & 0.029 & $54.26(1.52-1932.91)$ & 0.028 \\
\hline Acute renal failure & $24.50(4.49-133.76)$ & $<0.001$ & $54.31(1.52-1944.00)$ & 0.032 \\
\hline Positivity of urine TB PCR study & $8.94(1.01-79.05)$ & 0.030 & $5.56(0.19-162.56)$ & 0.320 \\
\hline Microscopic hematuria & $6.16(1.19-31.82)$ & 0.019 & $13.78(0.61-313.93)$ & 0.100 \\
\hline Proteinuria & $5.42(1.25-23.45)$ & 0.029 & $0.30(0.01-12.85)$ & 0.533 \\
\hline
\end{tabular}

Bold values indicate statistically significant differences

GUTB genitourinary tuberculosis, $O R$ odds ratio, $C l$ confidence interval, $T B$ tuberculosis, $P C R$ polymerase chain reaction 
infection of the kidney parenchyma or ureteral obstruction with resultant hydronephrosis.

In South Korea, GUTB comprises 3\% of EPTB cases. However, GUTB reportedly accounts for 27\% (range, 14$41 \%)$ of EPTB cases in the US, Canada and United Kingdom [4]. This difference may be due to the low prevalence $(<1 \%)$ of HIV infection in South Korea. In addition, South Korea has a higher incidence of TB than those of other countries and a high income level, but an intermediate incidence of TB. Thus, further research is needed.

There was no significant difference in the incidence of drug-related side effects, treatment duration or drug regimens between the two groups; thus, such drugs likely did not affect kidney function to an appreciable degree. In univariate analyses, urine TB positivity detected by PCR was associated with CKD. We presumed that the results of this test reflect disease severity and the amount of excretory bacilluria. Delayed diagnosis could aggravate destruction of the GU system, leading to CKD [10, 23]. Therefore, a more sensitive diagnostic method is needed. The diagnosis was confirmed most frequently by histopathology, but urine AFB staining for M. tuberculosis was the first-choice diagnostic method in almost all of the patients. Similar to previous reports, the sensitivity of urine AFB testing was very low $[21,23]$.

Of the patients, $75.0 \%$ underwent surgery, similar to previous reports $[5,18]$. Furthermore, the CKD group had a significantly higher incidence of additional surgical procedures and ESRD during follow up. Therefore, prevention of CKD development would reduce the need for unnecessary surgical procedures and dialysis. This will require considerable efforts to ensure early diagnosis of GUTB, including the development of a highly sensitive diagnostic tool and active testing of patients.

This study had several limitations. The first was its retrospective design. Second, some patients were lost to follow up, and some had been diagnosed and started treatment at another hospital and were subsequently transferred to our hospital. Third, 14 patients $(20 \%)$ were excluded because of insufficient information. Fourth, proteinuria was detected by the dipstick test, which is not quantitative. Fifth, the single-centre design and small sample size may limit the generalisability of our findings to the overall population. However, previous literature reviews comprise mainly case reports or hypothesised that CKD is related to GUTB. Thus, this study is the first to analyse the risk factors for CKD in GUTB patients.

\section{Conclusions}

ARF and old age are independent risk factors for CKD in GUTB patients. Therefore, in elderly GUTB patients with an elevated creatinine level at the time of diagnosis, regular follow-up of renal function should be performed during and after treatment.

\section{Abbreviations}

AFB: Acid-fast bacilli; ARF: Acute renal failure; BMl: Body mass index; Cl: Confidence interval; CKD: Chronic kidney disease; eGFR: estimated glomerular filtration rate; EPTB: Extrapulmonary tuberculosis; ESRD: End-stage renal disease; GU: Genitourinary; GUTB: Genitourinary tuberculosis; HIV: Human immunodeficiency virus; IQR: Interquartile range; KDIGO: Kidney Disease: Improving Global Outcomes; M. tuberculosis: Mycobacterium tuberculosis; MDRD: Modification of Diet in Renal Disease study equation; OR: Odds ratio; PCR: Polymerase chain reaction; TB: Tuberculosis

\section{Funding}

This work was supported by a National Research Foundation of Korea (NRF) grant funded by the Korean government (MSIT) (2017R1C1B5017875). This study was also supported by a faculty research grant from University College of Medicine (6-2017-0054). The fund provided data collection, statistical analysis and English proofing.

\section{Availability of data and materials}

The datasets used in this study are available from the corresponding author upon reasonable request.

\section{Authors' contributions}

Conception and design of study: EJK, NSK and YHC. Acquisition of data: EJK, WJL, WYJ, HC and IYJ. Data analysis and interpretation: EJK, NSK and YHC. Drafting of manuscript and critical revision: EJK, JYA, SJJ, NSK, JYC, YHC, YGS, JMK. Approval of final version of manusciript: EJK, WJL, WYJ, HC, IYJ, JYA, SJJ, NSK, JYC, YHC, YGS, and JMK.

\section{Ethics approval and consent to participate}

The study was approved by the Institutional Review Board of the Yonsei University Health System Clinical Trial Center. The requirement for informed consent was waived because this was a retrospective study involving a review of electronic medical records.

\section{Consent for publication \\ Not applicable.}

\section{Competing interests}

The authors declare that they have no competing interests.

\section{Publisher's Note}

Springer Nature remains neutral with regard to jurisdictional claims in published maps and institutional affiliations.

\section{Author details}

${ }^{1}$ Department of Infectious Diseases, Ajou University School of Medicine, 164, World cup-ro, Yeongtong-gu, Suwon-si, Gyeonggi-do 16499, Republic of Korea. ${ }^{2}$ Division of Infectious Disease, Department of Internal Medicine, Yonsei University College of Medicine, 50-1 Yonsei-ro, Seodaemun-gu, Seoul 120-752, Republic of Korea. ${ }^{3}$ AIDS Research Institute, Yonsei University College of Medicine, Seoul, Republic of Korea.

Received: 22 January 2018 Accepted: 26 July 2018

Published online: 02 August 2018

\section{References}

1. Mitchell BG, Ferguson JK, Anderson M, Sear J, Barnett A. Length of stay and mortality associated with healthcare-associated urinary tract infections: a multi-state model. J Hosp Infect. 2016;93:92-9.

2. Selik RM, Byers RH Jr, Dworkin MS. Trends in diseases reported on U.S. death certificates that mentioned HIV infection, 1987-1999. J Acquir Immune Defic Syndr. 2002;29:378-87.

3. Dudeck MA, Edwards JR, Allen-Bridson K, Gross C, Malpiedi PJ, Peterson KD, et al. National Healthcare Safety Network report, data summary for 2013, device-associated module. Am J Infect Control. 2015;43:206-21.

4. Abbara A, Davidson RN. Etiology and management of genitourinary tuberculosis. Nat Rev Urol. 2011;8:678-88.

5. Figueiredo AA, Lucon AM. Urogenital tuberculosis: update and review of 8961 cases from the world literature. Rev Urol. 2008;10:207-17. 
6. Gupta S, Santhosh R, Meitei KS, Singh SR. Primary genito-urinary tuberculosis with bilateral urolithiasis and renal failure-an unusual case. J Clin Diagn Res. 2013;7:927-9.

7. Stunell H, Buckley O, Feeney J, Geoghegan T, Browne RF, Torreggiani WC. Imaging of acute pyelonephritis in the adult. Eur Radiol. 2007;17:1820-8.

8. ESRD Registry Committee, Korean Society of Nephrology. Current renal replacement thrapy in Korea - Insan memorial Dialysis registry, 2015: Korean Society of Nephrology; 2016. http://www.ksn.or.kr/rang_board/list. html? code=sinchart

9. Kapoor R, Ansari MS, Mandhani A, Gulia A. Clinical presentation and diagnostic approach in cases of genitourinary tuberculosis. Indian J Urol. 2008;24:401-5.

10. Altiparmak MR, Trabulus S, Balkan II, Yalin SF, Denizli N, Aslan G, et al. Urinary tuberculosis: a cohort of 79 adult cases. Ren Fail. 2015;37:1157-63.

11. Levey AS, Bosch JP, Lewis JB, Greene T, Rogers N, Roth D. A more accurate method to estimate glomerular filtration rate from serum creatinine: a new prediction equation. Modification of diet in renal disease study group. Ann Intern Med. 1999;130:461-70.

12. Locatelli F, Nissenson AR, Barrett BJ, Walker RG, Wheeler DC, Eckardt KU, et al. Clinical practice guidelines for anemia in chronic kidney disease: problems and solutions. A position statement from kidney disease: improving global outcomes (KDIGO). Kidney Int. 2008;74:1237-40.

13. Murray CJ, Lopez AD. Measuring the global burden of disease. N Engl J Med. 2013;369:448-57.

14. Coresh J, Selvin E, Stevens LA, Manzi J, Kusek JW, Eggers P, et al. Prevalence of chronic kidney disease in the United States. JAMA. 2007;298:2038-47.

15. Zhang L, Wang F, Wang L, Wang W, Liu B, Liu J, et al. Prevalence of chronic kidney disease in China: a cross-sectional survey. Lancet. 2012;379:815-22.

16. Kim S, Lim CS, Han DC, Kim GS, Chin HJ, Kim SJ, et al. The prevalence of chronic kidney disease (CKD) and the associated factors to CKD in urban Korea: a population-based cross-sectional epidemiologic study. J Korean Med Sci. 2009;24(Suppl):S11-21.

17. Park Jl, Baek H, Jung HH. Prevalence of chronic kidney disease in Korea: the Korean National Health and nutritional examination survey 2011-2013. J Korean Med Sci. 2016:31:915-23.

18. Lee JY, Park HY, Park SY, Lee SW, Moon HS, Kim YT, et al. Clinical characteristics of genitourinary tuberculosis during a recent 10-year period in one center. Korean J Urol. 2011;52:200-5.

19. Tonelli M, Riella MC. World kidney day 2014: CKD and the aging population. Am J Kidney Dis. 2014;63:349-53.

20. Bolignano D, Mattace-Raso F, Sijbrands EJ, Zoccali C. The aging kidney revisited: a systematic review. Ageing Res Rev. 2014;14:65-80.

21. Daher Ede F, da Silva GB Jr, Barros EJ. Renal tuberculosis in the modern era. Am J Trop Med Hyg. 2013;88:54-64.

22. Figueiredo AA, Lucon AM, Srougi M. Urogenital tuberculosis. Microbiol Spectr. 2017;5 https://doi.org/10.1128/microbiolspec.TNM17-0015-2016.

23. Wang J, Fan S, Xiao J, Liang CZ. Renal tuberculosis tends to be low symptoms: how to improve the diagnosis and treatment of renal tuberculosis. Asian J Androl. 2016;18:145-6.

Ready to submit your research? Choose BMC and benefit from:

- fast, convenient online submission

- thorough peer review by experienced researchers in your field

- rapid publication on acceptance

- support for research data, including large and complex data types

- gold Open Access which fosters wider collaboration and increased citations

- maximum visibility for your research: over $100 \mathrm{M}$ website views per year

At BMC, research is always in progress.

Learn more biomedcentral.com/submissions 